Pacific

Journal of

Mathematics

\title{
LINES OF MINIMA ARE UNIFORMLY QUASIGEODESIC
}

Young-Eun Choi, KasRa RAFI AND CAROLINE SERIES 


\title{
LINES OF MINIMA ARE UNIFORMLY QUASIGEODESIC
}

\author{
Young-Eun Choi, KasRa Rafi And CARoline Series
}

\begin{abstract}
We continue the comparison between lines of minima and Teichmüller geodesics begun in our previous work. For two measured laminations $v^{+}$and $v^{-}$that fill up a hyperbolizable surface $S$ and for $t \in(-\infty, \infty)$, let $\mathscr{L}_{t}$ be the unique hyperbolic surface that minimizes the length function $e^{t} l\left(v^{+}\right)+$ $e^{-t} l\left(v^{-}\right)$on Teichmüller space. We prove that the path $t \mapsto \mathscr{L}_{t}$ is a Teichmüller quasigeodesic.
\end{abstract}

\section{Introduction}

This paper continues the comparison between lines of minima and Teichmüller geodesics begun in [Choi et al. 2006]. Let $S$ be a hyperbolizable surface of finite type and $\mathscr{T}(S)$ be the Teichmüller space of $S$. Let $v^{+}$and $v^{-}$be two measured laminations that fill up $S$. The associated line of minima is the path $t \mapsto \mathscr{L}_{t} \in \mathscr{T}(S)$, where $\mathscr{L}_{t}=\mathscr{L}_{t}\left(v^{+}, v^{-}\right)$is the unique hyperbolic surface that minimizes the length function $e^{t} l\left(v^{+}\right)+e^{-t} l\left(v^{-}\right)$on $\mathscr{T}(S)$; see [Kerckhoff 1992] and Section 2 below. Lines of minima have significance for hyperbolic 3-manifolds: infinitesimally bending $\mathscr{L}_{t}$ along the lamination $v^{+}$results in a quasifuchsian group whose convex core boundary has bending measures in the projective classes $v^{+}$and $v^{-}$and in the ratio $e^{2 t}: 1$; see [Series 2005]. In this paper we prove:

Theorem A. The line of minima $\mathscr{L}_{t}$ for $t \in \mathbb{R}$ is a quasigeodesic with respect to the Teichmüller metric. In other words, there are universal constants $c>1$ and $C>0$, depending only on the topology of $S$, such that for any $a, b \in \mathbb{R}$ with $a<b$, we have

$$
\frac{b-a}{c}-C \leq d_{\mathscr{T}(S)}\left(\mathscr{L}_{a}, \mathscr{L}_{b}\right) \leq c(b-a)+C,
$$

where $d_{T_{(S)}}$ is the Teichmüller distance.

An obvious way to approach this would be to compare the time- $t$ surface $\mathscr{L}_{t}$ with the corresponding surface $\varphi_{t}$ on the Teichmüller geodesic whose horizontal and vertical foliations at time $t$ are respectively, $e^{t} v^{+}$and $e^{-t} v^{-}$[Gardiner and Masur 1991]. In [Choi et al. 2006], we did just this. We showed that if neither surface $\mathscr{L}_{t}$ nor $\mathscr{G}_{t}$ contains short curves, that is, they are both contained in the thick

MSC2000: $30 \mathrm{~F} 60$.

Keywords: lines of minima, Teichmüller space, quasigeodesic. 
part of Teichmüller space, then the Teichmüller distance between them is bounded above by a uniform constant that is independent of $t$. More generally, we showed that the set of curves which are short on the two surfaces coincide. We also showed, however, that the ratio of lengths of the same short curve on the two surfaces may be arbitrarily large so the path $\mathscr{L}_{t}$ may deviate arbitrarily far from $\mathscr{G}_{t}$. It is therefore not immediately obvious how to derive Theorem A from [Choi et al. 2006]. To explain our method, we first summarize the results of [Choi et al. 2006] in more detail.

It turns out that on both $\mathscr{L}_{t}$ and $\mathscr{G}_{t}$, a curve $\alpha$ is short if and only if at least one of two quantities $D_{t}(\alpha)$ and $K_{t}(\alpha)$ is large. These quantities depend on the topological relationship between $\alpha$ and the defining laminations $v^{+}$and $v^{-}$. They relate to the modulus of a maximal embedded annulus around $\alpha$; the modulus of a flat annulus is approximately $D_{t}(\alpha)$ and the modulus of an expanding annulus is approximately $\log K_{t}(\alpha)$; see [Minsky 1992] and Sections 2 and 3 below. We say that a curve is extremely short if it is less than some prescribed $\epsilon_{0}>0$ depending only on the topology of $S$; see Section 2. The essential results in [Choi et al. 2006] were the following estimates (see Section 2 for notation).

Theorem 1.1 [Choi et al. 2006, Theorems 5.10, 5.13, 7.13, 7.14]. Let $\alpha$ be a simple closed curve on $S$. If $\alpha$ is extremely short on $\varphi_{t}$ then

$$
\frac{1}{l_{\varphi_{t}}(\alpha)} \asymp \max \left\{D_{t}(\alpha), \log K_{t}(\alpha)\right\},
$$

while if $\alpha$ is extremely short on $\mathscr{L}_{t}$ then

$$
\frac{1}{l_{\mathscr{L}_{t}}(\alpha)} \asymp \max \left\{D_{t}(\alpha), \sqrt{K_{t}(\alpha)}\right\} .
$$

Theorem 1.2 [Choi et al. 2006, Theorem 7.15]. The Teichmüller distance between $\mathscr{L}_{t}$ and $\mathscr{G}_{t}$ is given by

$$
d_{\mathscr{T}(S)}\left(\mathscr{L}_{t}, \mathscr{G}_{t}\right) \doteq \frac{1}{2} \log \max _{\alpha}\left\{\frac{l_{\mathscr{G}_{t}}(\alpha)}{l_{\mathscr{L}_{t}}(\alpha)}\right\},
$$

where the maximum is taken over all simple closed curves $\alpha$ that are extremely short in $\mathscr{G}_{t}$. In particular, the distance between the thick parts of $\mathscr{L}_{t}$ and $\mathscr{G}_{t}$ is bounded.

It follows from these results that, along intervals on which either there are no short curves or $D_{t}(\alpha)$ dominates for all short curves $\alpha$, the surfaces $\mathscr{L}_{t}$ and $\mathscr{G}_{t}$ remain a bounded distance apart. However the path $\mathscr{L}_{t}$ may deviate arbitrarily far from $\mathscr{G}_{t}$ along time intervals on which $K_{t}(\alpha)$ is large and dominates $D_{t}(\alpha)$. The situation is complicated by the fact that as we move along $\mathscr{L}_{t}$, the family of curves 
which are short at a given point in time will vary with $t$, so the intervals along which different curves $\alpha$ are short will overlap.

In addition to the above results from [Choi et al. 2006], there are two main ingredients in the proof of Theorem A. The first is a detailed comparison of the rates of change of $K_{t}(\alpha)$ and $D_{t}(\alpha)$ with $t$. Some simple estimates are made in Lemmas 3.1 and 3.2, with more elaborate consequences drawn in Lemma 5.1 and especially Lemma 5.2. These results use Minsky's product regions theorem (see Theorem 2.4), which allows us to reduce calculations of distance in regions of Teichmüller space in which a given family of curves is short, to straightforward estimates in $\mathbb{H}^{2}$. To apply Minsky's theorem, we need to compare not only lengths but also twists. We rely on the bounds on twists proved in [Choi et al. 2006] and reviewed in Theorems 2.8 and 2.9; these enter in a crucial way into the proof of Lemma 5.1.

The second main ingredient is control of distance along intervals along which $K_{t}(\alpha)$ is large. Consider the surface $S_{\alpha}$ obtained by cutting $S$ along a short curve $\alpha$ and replacing the two resulting boundary components by punctures. The following rather surprising result, proved in Section 4, states that on intervals along which $K_{t}(\alpha)$ is large, we can estimate the Teichmüller distance by restricting to the Teichmüller space of the surface $S_{\alpha}$. In other words, the contribution to Teichmüller distance in Minsky's formula 2.4 due to the short curve $\alpha$ itself may be neglected; see Theorem 4.1 for a precise statement.

Theorem B. If $K_{t}(\alpha)$ is sufficiently large for all $t \in[a, b]$, the distance in $\mathscr{T}\left(S_{\alpha}\right)$ between the restrictions of $\mathscr{G}_{a}$ and $\varphi_{b}$ to $S_{\alpha}$ is equal to $b-a$, up to an additive error that is bounded by a constant depending only on the topology of $S$.

The proof of Theorem A requires estimating upper and lower bounds for

$$
d_{\mathscr{T}(S)}\left(\mathscr{L}_{a}, \mathscr{L}_{b}\right)
$$

over very large time intervals $[a, b]$. Given the first of the two ingredients above, the upper bound is relatively straightforward. The lower bound depends on Theorem B. The actual application involves a rather subtle inductive procedure based on Lemma 5.2 which shows that at least one term in Minsky's formula 2.4 always involves a contribution comparable to $b-a$.

The paper is organized as follows. Section 2 gives standard background and introduces the twist $\mathrm{t} w_{\sigma}(\xi, \alpha)$ of a lamination $\xi$ about a curve $\alpha$ with respect to a hyperbolic metric $\sigma$. We give the main estimates about twists from [Choi et al. 2006]. In Section 3, we recall from [Choi et al. 2006] the definitions of $D_{t}(\alpha)$ and $K_{t}(\alpha)$ and derive some elementary results about their rates of change with $t$. In Section 4 we prove Theorem B and in Section 5 we prove Theorem A. 


\section{Background}

Notation. Since we will be dealing mainly with coarse estimates, we want to avoid heavy notation and keep track of constants which are universal, in that they do not depend on any specific metric or curve under discussion. For functions $f, g$ we write $f \asymp g$ to mean that there are constants $c \geq 1, C \geq 0$, depending only on the topology of $S$ and the fixed constant $\epsilon_{0}$ (see below), such that

$$
\frac{1}{c} g(x)-C \leq f(x) \leq \operatorname{cg}(x)+C .
$$

We use

$$
f \stackrel{*}{\succcurlyeq} g \text { and } f \stackrel{+}{\asymp} g
$$

to mean that these inequalities hold with $C=0$ and $c=1$, respectively. The

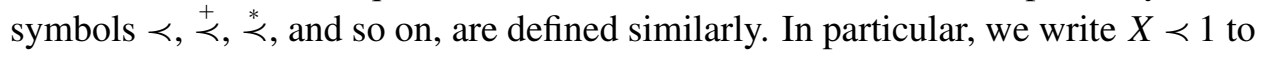
indicate $X$ is bounded above by a positive constant depending only on the topology of $S$ and $\epsilon_{0}$.

Short curves. Let $\mathscr{C}(S)$ denote the set of isotopy classes of nontrivial, nonperipheral simple closed curves on $S$. The length of the geodesic representative of $\alpha \in \mathscr{C}(S)$ with respect to a hyperbolic metric $\sigma \in \mathscr{T}(S)$ will be denoted $l_{\sigma}(\alpha)$. In our dealings with short curves we will have to make various assumptions to ensure the validity of our estimates, which all require that the length $l_{\sigma}(\alpha)$ of a "short" curve is less than various constants, in particular less than the Margulis constant. We suppose that $\epsilon_{0}>0$ is chosen once and for all to satisfy all needed assumptions, and say a simple closed curve $\alpha$ is extremely short in $\sigma$ if $l_{\sigma}(\alpha)<\epsilon_{0}$.

Measured laminations and Teichmuiller space. We denote the space of measured laminations on $S$ by $\mathcal{M L}(S)$ and write $l_{\sigma}(\xi)$ for the hyperbolic length of a measured lamination $\xi \in \mathcal{M L}(S)$. For $\xi \in \mathcal{M L}(S)$, we denote the underlying leaves by $|\xi|$.

Kerckhoff lines of minima. Suppose that $v^{+}, v^{-} \in M \mathscr{L}(S)$ fill up $S$, meaning that the sum of (geometric) intersections $i\left(v^{+}, \xi\right)+i\left(v^{-}, \xi\right)>0$ for all $\xi \in M \mathscr{L}(S)$. Kerckhoff [1992] showed that the sum of length functions

$$
\sigma \mapsto l_{\sigma}\left(v^{+}\right)+l_{\sigma}\left(v^{-}\right)
$$

has a unique global minimum on $\mathscr{T}(S)$. Moreover, as $t$ varies in $(-\infty, \infty)$, the minimum $\mathscr{L}_{t} \in \mathcal{T}(S)$ of $l\left(v_{t}^{+}\right)+l\left(v_{t}^{-}\right)$for the measured laminations $v_{t}^{+}=e^{t} v^{+}$ and $v_{t}^{-}=e^{-t} v^{-}$varies continuously with $t$ and traces out a path $t \mapsto \mathscr{L}_{t}$ called the line of minima $\mathscr{L}\left(v^{+}, v^{-}\right)$of $v^{ \pm}$.

Teichmüller geodesics. A pair of laminations $v^{+}, v^{-} \in \mathcal{M L}(S)$ which fill up $S$ also defines a Teichmüller geodesic $\mathscr{G}=\mathscr{G}\left(v^{+}, v^{-}\right)$. The time- $t$ surface $\mathscr{G}_{t} \in \mathscr{G}$ is the 
unique Riemann surface that supports a quadratic differential $q_{t}$ whose horizontal and vertical foliations are the measured foliations corresponding to $v_{t}^{+}$and $v_{t}^{-}$ respectively; see [Gardiner and Masur 1991; Levitt 1983]. Flowing distance $d$ along $\mathscr{G}$ expands the vertical foliation by a factor $e^{d}$ and contracts the horizontal foliation by $e^{-d}$. By abuse of notation, we denote the hyperbolic metric on the surface $\mathscr{G}_{t}$ also by $\mathscr{G}_{t}$, and likewise denote the quadratic differential metric defined by $q_{t}$ also by $q_{t}$.

Balance time. For a curve $\alpha \in \mathscr{C}(S)$ that is not a component of either the vertical or the horizontal foliation, let $t_{\alpha}$ denote the balance time of $\alpha$ at which

$$
i\left(\alpha, v_{t}^{+}\right)=i\left(\alpha, v_{t}^{-}\right) .
$$

Along $\mathscr{G}$, a curve is shortest near its balance time. More precisely, we have the following proposition, which follows from [Rafi 2007, Theorem 3.1]:

Proposition 2.1. Choose $\epsilon>0$ so that $\epsilon<\epsilon_{0}$ and suppose that $l_{\varphi_{t_{\alpha}}}(\alpha)<\epsilon$. Let $I_{\alpha}=I_{\alpha}(\epsilon)$ be the maximal connected interval containing $t_{\alpha}$ such that $l_{\varphi_{t}}(\alpha)<\epsilon$ for all $t \in I_{\alpha}$. Then there is a constant $\epsilon^{\prime}>0$ depending only on $\epsilon$ such that $l_{\varphi_{t}}(\alpha) \geq \epsilon^{\prime}$ for all $t \notin I_{\alpha}$. (If $l_{\varphi_{t_{\alpha}}}(\alpha) \geq \epsilon$ then set $I_{\alpha}=\varnothing$.)

Curves which are components of the vertical foliation $\left(i\left(\alpha, v^{-}\right)=0\right.$, called vertical) or the horizontal foliation $\left(i\left(\alpha, v^{+}\right)=0\right.$, called horizontal $)$ are exceptional but in general easier to handle. In such cases, $t_{\alpha}$ is undefined. However, for reasons of continuity, it is natural to adopt the convention that when $\alpha$ is vertical $t_{\alpha}=$ $-\infty$ and when $\alpha$ is horizontal $t_{\alpha}=\infty$. Moreover, the arguments used to prove Proposition 2.1 still hold; when $\alpha$ is vertical (respectively, horizontal), we define

$$
I_{\alpha}=(-\infty, c) \quad\left(\text { respectively, } I_{\alpha}=(d, \infty)\right)
$$

to be the maximal interval where $l \varphi_{t}(\alpha)<\epsilon$.

Flat and expanding annuli. Let $\sigma$ be a hyperbolic metric and let $q$ be any quadratic differential metric in the same conformal class. Let $A$ be an annulus in $(S, q)$ with piecewise smooth boundary. The following notions are due to Minsky [1992]. We say $A$ is regular if the boundary components $\partial_{0}, \partial_{1}$ are equidistant from one another and the curvature along $\partial_{0}, \partial_{1}$ is either nonpositive at every point or nonnegative at every point (see [Minsky 1992] or [Choi et al. 2006] for details). We follow the sign convention that the curvature at a smooth point of $\partial A$ is positive if the acceleration vector points into $A$. Suppose $A$ is a regular annulus such that the total curvature of $\partial_{0}$ satisfies $\kappa\left(\partial_{0}\right) \leq 0$. Then, it follows from the Gauss-Bonnet theorem that $\kappa\left(\partial_{1}\right) \geq 0$. We say $A$ is flat if $\kappa\left(\partial_{0}\right)=\kappa\left(\partial_{1}\right)=0$ and say $A$ is expanding if $\kappa\left(\partial_{0}\right)<0$, and call $\partial_{0}$ the inner boundary and $\partial_{1}$ the outer boundary. 
A regular annulus is primitive if it contains no singularities of $q$ in its interior. It follows that a flat annulus is primitive and is isometric to a cylinder obtained from a Euclidean rectangle by identifying one pair of parallel sides. An expanding annulus that is primitive is coarsely isometric to an annulus bounded by two concentric circles in the plane.

The length of a curve $\alpha$ which is short in $(S, \sigma)$ can be estimated by the modulus of a primitive annulus around it:

Theorem 2.2 [Minsky 1992, Theorem 4.5; Choi et al. 2006, Theorem 5.3]. Suppose $\alpha \in \mathscr{C}(S)$ is extremely short in $(S, \sigma)$. Then for any quadratic differential metric $q$ in the same conformal class as $\sigma$, there is an annulus $A$ that is primitive with respect to $q$ whose core is homotopic to $\alpha$ such that

$$
\frac{1}{l_{\sigma}(\alpha)} \asymp \operatorname{Mod}(A) .
$$

The modulus of a primitive annulus is estimated as follows:

Theorem 2.3 [Minsky 1992, Theorem 4.5; Rafi 2005, Lemma 3.6]. Let $A \subset S$ be a primitive annulus. Let $d$ be the $q$-distance between the boundary components $\partial_{0}$, $\partial_{1}$. If $A$ is expanding let $\partial_{0}$ be the inner boundary. Then either

(i) $A$ is flat and $\operatorname{Mod} A=d / l_{q}\left(\partial_{0}\right)=d / l_{q}\left(\partial_{1}\right)$ or

(ii) $A$ is expanding and $\operatorname{Mod} A \asymp \log \left(d / l_{q}\left(\partial_{0}\right)\right)$.

Minsky's product regions theorem. Our main tool for estimating Teichmüller distance is Minsky's product regions theorem, which reduces the estimation of the distance between two surfaces on which a given set $\Gamma$ of curves is short to a calculation in the hyperbolic plane $\mathbb{H}^{2}$. To give a precise statement, we introduce the following notation. Choose a pants curves system on $S$ that contains $\Gamma$, and for a curve $\alpha$ in the pants system let $s_{\alpha}(\sigma)$ be the Fenchel-Nielsen twist coordinate of $\alpha$. (Here $s_{\alpha}(\sigma)=\tilde{s}_{\alpha}(\sigma) / l_{\sigma}(\alpha)$, where $\tilde{s}_{\alpha}(\sigma)$ is the actual hyperbolic distance twisted round $\alpha$; see Minsky [1996] for details.) Let $\mathscr{T}_{\text {thin }}\left(\Gamma, \epsilon_{0}\right)$ be the subset of $\mathscr{T}(S)$ on which all the curves in $\Gamma$ have length less than $\epsilon_{0}$ and let $S_{\Gamma}$ be the analytically finite surface obtained from $S$ by pinching all the curves in $\Gamma$. By forgetting the Fenchel-Nielsen length and twist coordinates associated to the curves in $\Gamma$ but retaining all remaining Fenchel-Nielsen coordinates, we obtain a projection

$$
\Pi_{\Gamma}: \mathscr{T}(S) \rightarrow \mathscr{T}\left(S_{\Gamma}\right) .
$$

For each $\alpha \in \Gamma$, let $\mathbb{\boxplus}_{\alpha}$ denote a copy of the upper-half plane and let $d_{\mathbb{H}_{\alpha}}$ denote half the usual hyperbolic metric on $\mathbb{H}_{\alpha}$ (see [Minsky 1996, Lemma 2.2] for the factor). Define $\Pi_{\alpha}: \mathscr{T}(S) \rightarrow \mathbb{H}_{\alpha}$ by $\Pi_{\alpha}(\sigma)=s_{\alpha}(\sigma)+i / l_{\sigma}(\alpha) \in \mathbb{H}_{\alpha}$. Then the product regions theorem states: 
Theorem 2.4 [Minsky 1996]. Let $\sigma, \tau \in \mathscr{T}_{\text {thin }}\left(\Gamma, \epsilon_{0}\right)$. Then

$$
d_{\mathscr{T}(S)}(\sigma, \tau) \doteq \max _{\alpha \in \Gamma}\left\{d_{\mathcal{T}_{\left(S_{\Gamma}\right)}}\left(\Pi_{\Gamma}(\sigma), \Pi_{\Gamma}(\tau)\right), d_{\mathbb{\boxplus}_{\alpha}}\left(\Pi_{\alpha}(\sigma), \Pi_{\alpha}(\tau)\right)\right\} .
$$

To simplify notation, we write

$$
d_{\mathbb{\boxplus}_{\alpha}}(\sigma, \tau)
$$

instead of $d_{\mathbb{\boxplus}_{\alpha}}\left(\Pi_{\alpha}(\sigma), \Pi_{\alpha}(\tau)\right)$ and

$$
d_{\mathcal{T}_{\left(S_{\Gamma}\right)}}(\sigma, \tau)
$$

instead of $d_{\mathcal{T}\left(S_{\Gamma}\right)}\left(\Pi_{\Gamma}(\sigma), \Pi_{\Gamma}(\tau)\right)$.

In practice, we usually apply Minsky's theorem with the aid of the following estimate from geometry in $\mathbb{\boxplus}^{2}$. The hyperbolic distance between two points $z_{1}, z_{2}$ in $\mathbb{H}^{2}$ is given by

$$
\cosh 2 d_{\mathbb{U}}\left(z_{1}, z_{2}\right)=1+\frac{\left|z_{1}-z_{2}\right|^{2}}{2 \operatorname{Im} z_{1} \operatorname{Im} z_{2}} .
$$

Let $\sigma_{a}, \sigma_{b}$ be two points in $\operatorname{Teich}(S)$ at which a curve $\alpha$ is short. Let $\ell_{a}, \ell_{b}$ and $s_{a}, s_{b}$ denote the Fenchel-Nielsen twist coordinate of $\alpha$ at $\sigma_{a}, \sigma_{b}$ respectively. It follows easily from the above formula that

$$
d_{\mathbb{\mathbb { M } _ { \alpha } ^ { 2 }}}\left(\sigma_{a}, \sigma_{b}\right) \doteq \frac{1}{2} \log \max \left\{\left|s_{a}-s_{b}\right|^{2} \ell_{a} \ell_{b}, \frac{\ell_{a}}{\ell_{b}}, \frac{\ell_{b}}{\ell_{a}}\right\} .
$$

Twists. Our estimates also require taking account of the $t w i s t \mathrm{t} w_{\sigma}(v, \alpha)$ of a lamination $v$ round $\alpha$ with respect to a hyperbolic metric $\sigma$. Following Minsky, we define

$$
\mathrm{t} w_{\sigma}(v, \alpha)=\inf \frac{\tilde{s}}{l_{\sigma}(\alpha)},
$$

where $\tilde{s}$ is the signed hyperbolic distance between the perpendicular projections of the endpoints of a lift of a geodesic in $|\nu|$ at infinity onto a lift of $\alpha$, and the infimum is over all lifts of leaves of $|\nu|$ which intersect $\alpha$; see [Minsky 1996] or [Choi et al. 2006] for details. We write $\mathrm{T} w_{\sigma}(v, \alpha)$ for $\left|\mathrm{t} w_{\sigma}(v, \alpha)\right|$. Notice that the twist $\mathrm{t} w_{\sigma}(v, \alpha)$ does not depend on the measure on $v$, but only on the underlying lamination $|v|$.

The twist is closely related to the Fenchel-Nielsen twist coordinate. Specifically, we have:

Lemma 2.5 [Minsky 1996, Lemma 3.5]. For any lamination $v \in \mathcal{M L}(S)$ and any two metrics $\sigma, \sigma^{\prime} \in \mathscr{T}(S)$,

$$
\left|\left(\mathrm{t} w_{\sigma}(\nu, \alpha)-\mathrm{t} w_{\sigma^{\prime}}(\nu, \alpha)\right)-\left(s_{\alpha}(\sigma)-s_{\alpha}\left(\sigma^{\prime}\right)\right)\right| \leq 4 .
$$

Although $\mathrm{t} w_{\sigma}(\nu, \alpha)$ depends on the metric $\sigma$, for $v_{1}, v_{2} \in \mathcal{M L}(S)$, the difference

$$
\mathrm{t} w_{\sigma}\left(v_{1}, \alpha\right)-\mathrm{t} w_{\sigma}\left(v_{2}, \alpha\right)
$$


is independent of $\sigma$ up to a universal additive constant; see [Minsky 1996; Choi et al. 2006, Section 4]. This motivates the following definition:

Definition 2.6. For $\alpha \in \mathscr{C}(S)$ and $v_{1}, v_{2} \in \mathcal{M L}(S)$, the relative twist of $v_{1}$ and $v_{2}$ round $\alpha$ is

$$
d_{\alpha}\left(v_{1}, v_{2}\right)=\inf _{\sigma}\left|\mathrm{t} w_{\sigma}\left(v_{1}, \alpha\right)-\mathrm{t} w_{\sigma}\left(v_{2}, \alpha\right)\right|,
$$

where the infimum is taken over all hyperbolic metrics $\sigma \in \mathscr{T}(S)$.

(The relative twist $d_{\alpha}\left(v_{1}, v_{2}\right)$ agrees up to an additive constant with the definition of subsurface distance between the projections of $\left|v_{1}\right|$ and $\left|v_{2}\right|$ to the annular cover of $S$ with core $\alpha$, as defined in [Masur and Minsky 2000, Section 2.4] and used throughout [Rafi 2005; 2007].)

Rafi [2007] (see also [Choi et al. 2006, Section 5.4]) introduced a similar notion of the twist $\mathrm{t} w_{q}(v, \alpha)$ with respect to a quadratic differential metric $q$ compatible with $\sigma$ and proved the following result, which enters into the proof of Theorem 4.1:

Proposition 2.7 Rafi 2007, Theorem 4.3; Choi et al. 2006, Proposition 5.7. Suppose that $\sigma \in \mathscr{T}(S)$ is a hyperbolic metric and $q$ is a compatible quadratic differential metric. For any geodesic lamination $\xi$ intersecting $\alpha$, we have

$$
\left|\mathrm{t} w_{\sigma}(\xi, \alpha)-\mathrm{t} w_{q}(\xi, \alpha)\right| \prec \frac{1}{l_{\sigma}(\alpha)} .
$$

We shall also need the following important estimates of the twist which complement Theorem 1.1. If $\alpha$ is vertical or horizontal, $t_{\alpha}$ is defined using the convention discussed following Proposition 2.1.

Theorem 2.8 [Choi et al. 2006, Theorems 5.11, 5.13]. Let $\alpha$ be a simple closed curve on $S$. If $\alpha$ is extremely short on $\mathscr{G}_{t}$ then

$$
\begin{aligned}
& \mathrm{T} w \varphi_{t}\left(v^{+}, \alpha\right) \prec \frac{1}{l \varphi_{t}(\alpha)} \quad \text { if } t>t_{\alpha}, \\
& \mathrm{T} w \varphi_{t}\left(v^{-}, \alpha\right) \prec \frac{1}{l \varphi_{t}(\alpha)} \quad \text { if } t<t_{\alpha} .
\end{aligned}
$$

Theorem 2.9 [Choi et al. 2006, Theorems 6.2,6.9]. Let $\alpha$ be a simple closed curve on $S$. If $\alpha$ is extremely short on $\mathscr{L}_{t}$ then

$$
\begin{aligned}
& \mathrm{T} w_{\mathscr{L}_{t}}\left(v^{+}, \alpha\right) \prec \frac{1}{l_{\mathscr{L}_{t}}(\alpha)} \quad \text { if } t>t_{\alpha}, \\
& \mathrm{T} w_{\mathscr{L}_{t}}\left(v^{-}, \alpha\right) \prec \frac{1}{l_{\mathscr{L}_{t}}(\alpha)} \quad \text { if } t<t_{\alpha} .
\end{aligned}
$$




\section{The length estimates}

In this section we discuss the quantities $D_{t}(\alpha)$ and $K_{t}(\alpha)$ that appear in the length estimates in Theorem 1.1.

Let $q_{t}$ be the unit-area quadratic differential metric on $\varphi_{t}$ whose vertical and horizontal foliations are $e^{t} v^{+}$and $e^{-t} v^{-}$, respectively. In light of Theorems 2.2 and 2.3, to estimate the length of a curve $\alpha$ which is extremely short in $\varphi_{t}$, it is sufficient to estimate the modulus of a maximal flat or expanding annulus around $\alpha$ in $q_{t}$. The union of all $q_{t}$-geodesic representatives of $\alpha$ foliate a Euclidean cylinder $F_{t}(\alpha)$, which is the maximal flat annulus whose core is homotopic to $\alpha$. (The cylinder is degenerate if the representative of $\alpha$ is unique.) On either side of $F_{t}(\alpha)$ is attached a maximal expanding annulus. Let $E_{t}(\alpha)$ be the one of larger modulus. Up to coarse equivalence, $D_{t}(\alpha)$ will be the modulus of $F_{t}(\alpha)$ while $\log K_{t}(\alpha)$ will be the modulus of $E_{t}(\alpha)$.

The precise definition of $D_{t}(\alpha)$ is as follows. If $\alpha$ is not a component of $\left|v^{ \pm}\right|$, we define

$$
D_{t}(\alpha)=e^{-2\left|t-t_{\alpha}\right|} d_{\alpha}\left(v^{+}, v^{-}\right)
$$

where $d_{\alpha}\left(v^{+}, v^{-}\right)$is the relative twisting of $v^{+}$and $v^{-}$about $\alpha$ as defined above. If $\alpha$ is vertical, define

$$
D_{t}(\alpha)=e^{-2 t} \operatorname{Mod} F_{0}(\alpha)
$$

and if $\alpha$ is horizontal, define

$$
D_{t}(\alpha)=e^{2 t} \operatorname{Mod} F_{0}(\alpha),
$$

where $F_{0}(\alpha)$ is the annulus at time $t=0$.

The precise definition of $K_{t}(\alpha)$ is

$$
K_{t}(\alpha)=\frac{d_{q_{t}}}{l_{q_{t}}\left(\partial_{0}\right)},
$$

where $\partial_{0}$ is the inner boundary of $E_{t}(\alpha)$ and $d_{q_{t}}$ is the $q_{t}$-distance between the inner and outer boundaries of $E_{t}(\alpha)$.

The connection with the definition of $K_{t}(\alpha)$ in [Choi et al. 2006] and the reasons why $D_{t}(\alpha)$ and $K_{t}(\alpha)$ are coarsely the moduli of $F_{t}(\alpha)$ and $E_{t}(\alpha)$ respectively are explained at the end of this section. The estimate for $1 / l \varphi_{t}(\alpha)$ in Theorem 1.1 follows easily from the above definitions and Minsky's estimates. The estimate for $1 / l_{\mathscr{L}_{t}}(\alpha)$ in the same theorem required a lengthy separate analysis. The only features of these definitions which will concern us here are the estimates in Theorem 1.1 and the relative rates of change of $D_{t}(\alpha)$ and $K_{t}(\alpha)$ with time. 
The rate of change of $D_{t}(\alpha)$ and $K_{t}(\alpha)$. The rate of change of $D_{t}(\alpha)$ with time is immediate from (3-1). To estimate the rate of change of $K_{t}(\alpha)$, note that, since $E_{t}(\alpha)$ is maximal, $d_{q_{t}}$ in (3-2) is half the $q_{t}$-length of an essential arc from $\alpha$ to itself. Since the $q_{t}$-length of such an arc or a simple closed curve can increase or decrease at the rate of at most $e^{ \pm t}$, (3-2) implies that $\sqrt{K_{t}(\alpha)}$ changes (in the coarse sense) at a rate at most $e^{t}$. More precisely, if $K_{t}(\alpha)$ is sufficiently large for all $t \in[a, b]$, then

$$
e^{-2(b-a)} K_{b}(\alpha) \stackrel{*}{\prec} K_{a}(\alpha) \stackrel{*}{\prec} e^{2(b-a)} K_{b}(\alpha) .
$$

In combination with (3-1) and Theorem 1.1, it follows that the length of a short curve along $\mathscr{L}$ or $\mathscr{G}$ changes at rate at most $e^{2 t}$. More detailed control is given by the following two lemmas, which should be understood with our convention on $t_{\alpha}$ to include the case when $\alpha$ is vertical or horizontal. The first shows that $K_{t}(\alpha)$ decays as $t$ moves away from $t_{\alpha}$ while the second, illustrated schematically in Figure 1, compares rates of change of $D_{t}(\alpha)$ and $\sqrt{K_{t}(\alpha)}$.

Lemma 3.1. The function $K_{t}(\alpha)$ decays as $t$ moves away from $t_{\alpha}$. More precisely,

(i) if $t_{\alpha}<v<w$, then $K_{v}(\alpha) \stackrel{*}{\succ} K_{w}(\alpha)$;

(ii) if $v<w<t_{\alpha}$, then $K_{w}(\alpha) \stackrel{*}{\succ} K_{v}(\alpha)$.

Proof. Suppose first that $\alpha$ is not a component of $\left|v^{ \pm}\right|$. By [Choi and Rafi 2007, Lemma 2.1] (see also [Rafi 2007, Theorem 2.1]) we have

$$
l_{q_{t}}(\alpha) \triangleq e^{\left|t-t_{\alpha}\right|} l_{q_{t_{\alpha}}}(\alpha)
$$

for any $t \in \mathbb{R}$. On the other hand, the length of any curve or arc can increase or decrease by a factor of at most $e^{ \pm t}$. Hence, if $t_{\alpha}<v<w$, then

$$
K_{v}(\alpha)=\frac{d_{q_{v}}}{l_{q_{v}}(\alpha)} \stackrel{*}{`} \frac{d_{q_{v}}}{e^{(v-w)} l_{q_{w}}(\alpha)}=\frac{e^{(w-v)} d_{q_{v}}}{l_{q_{w}}(\alpha)} \geq \frac{d_{q_{w}}}{l_{q_{w}}(\alpha)}=K_{w}(\alpha) .
$$
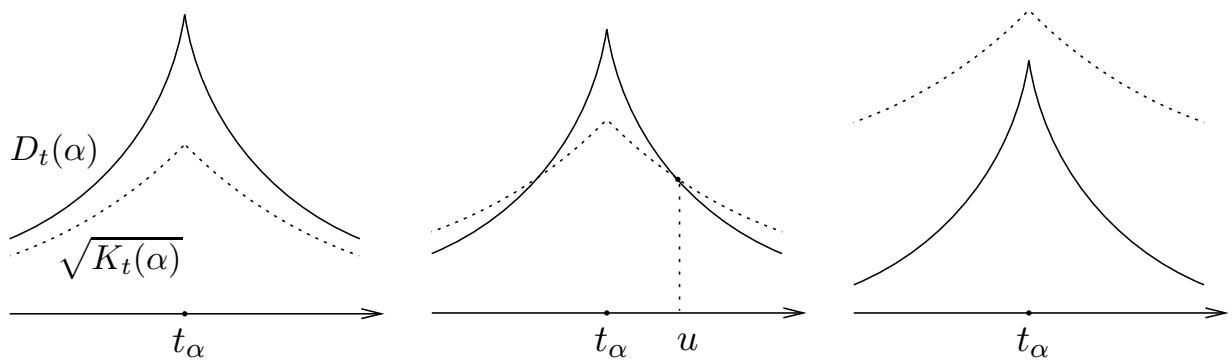

Figure 1. Schematic graphs of $D_{t}(\alpha)$ and $\sqrt{K_{t}(\alpha)}$. The function $D_{t}(\alpha)$ changes at rate $e^{2 t}$ while $\sqrt{K_{t}(\alpha)}$ changes at rate at most $e^{t}$. 
A similar argument can be applied in the case when $v<w<t_{\alpha}$.

If $\alpha$ is vertical, then

$$
l_{q_{t}}(\alpha) \stackrel{*}{\asymp} e^{t} l_{q_{0}}(\alpha),
$$

while if it is horizontal

$$
l_{q_{t}}(\alpha) \stackrel{*}{`} e^{-t} l_{q_{0}}(\alpha) .
$$

The result then follows in the same way.

Lemma 3.2. Let $I_{\alpha}$ be as in Proposition 2.1 and let $[a, b] \subset I_{\alpha}$. Suppose that $D_{u}(\alpha)=\sqrt{K_{u}(\alpha)}$ for some $u \in[a, b]$.

(i) If $t_{\alpha}<u$, then $\sqrt{K_{t}(\alpha)} \stackrel{*}{\succ} D_{t}(\alpha)$ for all $t \in[u, b]$.

(ii) If $u<t_{\alpha}$, then $\sqrt{K_{t}(\alpha)} \stackrel{*}{\succ} D_{t}(\alpha)$ for all $t \in[a, u]$.

Proof. We refer to Figure 1 for a schematic picture of the two graphs. The proof is based on the fact that $\sqrt{K_{t}(\alpha)}$ decays at a slower rate than $D_{t}(\alpha)$ as $t$ moves away from $t_{\alpha}$. If $t_{\alpha}<u$, then for any $t>u$ we have

$$
K_{t}(\alpha)=\frac{d_{q_{t}}}{l_{q_{t}}(\alpha)} \stackrel{*}{\succ} \frac{e^{-(t-u)} d_{q_{u}}}{e^{(t-u)} l_{q_{u}}(\alpha)}=e^{-2(t-u)} K_{u}(\alpha) .
$$

Therefore,

$$
\sqrt{K_{t}(\alpha)} \stackrel{*}{\succ} e^{-(t-u)} D_{u}(\alpha)=e^{(t-u)} D_{t}(\alpha) \geq D_{t}(\alpha) .
$$

A similar argument can be applied in the case when $u<t_{\alpha}$.

Alternative definitions of $D_{t}(\alpha)$ and $K_{t}(\alpha)$. The remarks which follow are not essential for the proof of Theorem A but may be helpful in clarifying background from [Choi et al. 2006].

The claim that $D_{t}(\alpha)$ is coarsely equal to the modulus of $F_{t}(\alpha)$ is justified by [Choi et al. 2006, Proposition 5.8 (Section 5.6 for the exceptional case)] which states that $\operatorname{Mod} F_{t}(\alpha) \asymp D_{t}(\alpha)$. The proof is an exercise in Euclidean geometry, combined with Rafi's comparison Proposition 2.7 between the twist in the quadratic and hyperbolic metrics. For example, at the balance time $t_{\alpha}$, the horizontal and vertical leaves both make an angle $\pi / 4$ with the $q_{t_{\alpha}}$-geodesic representatives of $\alpha$. Let $\eta$ be an arc joining the two boundary components of $F_{t_{\alpha}}$ that is orthogonal to all the $q_{t_{\alpha}}$-geodesic representatives of $\alpha$. In this case, a leaf of $v_{t_{\alpha}}^{+}$or $v_{t_{\alpha}}^{-}$intersects $\eta$ approximately (up to an error of 1) $l_{q_{t_{\alpha}}}(\eta) / l_{q_{t_{\alpha}}}(\alpha)$ times, so the modulus of $F_{t_{\alpha}}(\alpha)$ is approximated by

$$
\mathrm{T} w_{F_{t_{\alpha}}}\left(v^{+}, \alpha\right)=\mathrm{T} w_{F_{t_{\alpha}}}\left(v^{-}, \alpha\right),
$$

where $\mathrm{t} w_{F_{t_{\alpha}}}$ (and $\mathrm{T} w_{F_{t_{\alpha}}}$ ) means the twist in the $q$-metric restricted to $F_{t_{\alpha}}$. The result would follow by noting that $\mathrm{t} w_{F_{t_{\alpha}}}\left(v^{+}, \alpha\right)$ and $\mathrm{t} w_{F_{t_{\alpha}}}\left(v^{-}, \alpha\right)$ have opposite signs, except that $d_{\alpha}$ involves hyperbolic twists on $S$ rather than $q$-twists in $F_{t_{\alpha}}$. This is resolved using Proposition 2.7; see [Choi et al. 2006] for further details. 
That $\log K_{t}(\alpha)$ is coarsely the modulus of $E_{t}(\alpha)$ follows from Theorem 2.3. The above is not the definition of $K_{t}(\alpha)$ given in [Choi et al. 2006], but it is coarsely equivalent. Specifically, let $Y_{1}, Y_{2}$ be the (possibly coincident) thick components adjacent to $\alpha$ in the thick-thin decomposition of the hyperbolic metric $\mathscr{G}_{t}$. Set

$$
J_{t}(\alpha)=\frac{1}{l_{q_{t}}(\alpha)} \max \left\{\lambda_{Y_{1}}, \lambda_{Y_{2}}\right\}
$$

where $\lambda_{Y_{i}}$ is the length of the shortest nontrivial nonperipheral simple closed curve on $Y_{i}$ with respect to the metric $q_{t}$. (If either $Y_{i}$ is a pair of pants there is a slightly different definition; see [Choi et al. 2006].) In [Choi et al. 2006], we took the above expression for $J_{t}(\alpha)$ as the definition of $K_{t}(\alpha)$. [Choi et al. 2006, Proposition 5.9] shows that if $J_{t}(\alpha)$ is sufficiently large, then $J_{t}(\alpha) \stackrel{*}{`} K_{t}(\alpha)$ with $K_{t}(\alpha)$ defined as in (3-2) above.

\section{Expanding annuli that persist}

It follows from Theorems 1.1 and 1.2 that if $D_{t}(\alpha) \geq \sqrt{K_{t}(\alpha)}$ for every $\alpha$ that is short in $\mathscr{G}_{t}$, then the distance $d_{\mathscr{T}(S)}\left(\mathscr{G}_{t}, \mathscr{L}_{t}\right)$ is uniformly bounded. Moreover, if $\mathscr{G}_{t}$ is in the thick part of Teichmüller space, then $\mathscr{L}_{t}$ is too, so that on such intervals $\mathscr{L}_{t}$ is quasigeodesic. Thus our attention is focused on time intervals along which $K_{t}(\alpha)$ is large. This is handled with the following more precise version of Theorem B.

Theorem 4.1. Choose $M>0$ to be a constant such that if $K_{t}(\alpha)>M$ then $\alpha$ is extremely short in $\varphi_{t}$. (This is possible due to Theorem 1.1.) Suppose that $K_{t}(\alpha)>M$ for all $t \in[a, b]$. Then

$$
d \mathscr{T}_{\left(S_{\alpha}\right)}\left(\mathscr{G}_{a}, \mathscr{G}_{b}\right) \doteq b-a .
$$

Corollary 4.2. Let $\Gamma$ be a family of disjoint curves on $S$ such that $K_{t}(\alpha)>M$ for all $t \in[a, b]$ and for every $\alpha \in \Gamma$. Then

$$
d_{T_{\left(S_{\Gamma}\right)}}\left(\mathscr{G}_{a}, \mathscr{G}_{b}\right) \succsim b-a .
$$

Proof. We prove the statement of the theorem; the corollary is immediate. The idea is that for each $t \in[a, b]$, we cut the maximal flat annulus around $\alpha$ in $\left(S, q_{t}\right)$ out of $S$ and reglue the two boundary components, obtaining a new surface $\bar{\varphi}_{t}$; see Figure 2. The surfaces $\bar{G}_{t}$ will also move along a Teichmüller geodesic. In particular

$$
d_{\mathscr{T}(S)}\left(\overline{\mathscr{G}}_{a}, \overline{\mathscr{G}}_{b}\right)=b-a .
$$

On the other hand, $\overline{\mathscr{G}}_{t}$ contains the same expanding cylinders round $\alpha$ as $\mathscr{G}_{t}$ so that $K_{\bar{g}_{t}}(\alpha)=K_{t}(\alpha)$. Consideration of the rate of change of $K_{t}$ with time shows that the contribution to the change in Teichmüller distance between $\bar{\varphi}_{a}$ and $\bar{\varphi}_{b}$ from the 


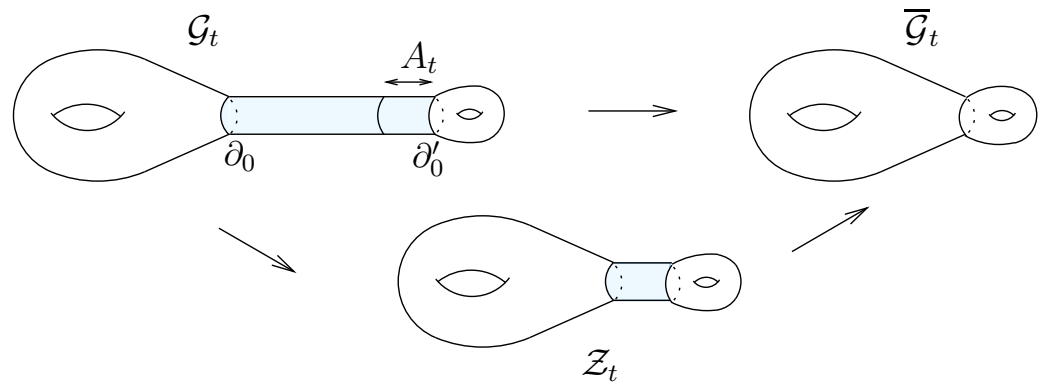

Figure 2. Cut out flat annulus and reglue.

expanding cylinders is of the order of $\log (b-a)$, so the actual distance $b-a$ must be realized due to changes in $\mathscr{T}\left(S_{\alpha}\right)$.

In more detail, this works as follows. Let $F=F_{a}(\alpha)$ be the maximal flat annulus around $\alpha$ in $\left(S, q_{a}\right)$. The arcs in $F$ that are perpendicular to $\partial F$ define an isometry $f$ from one component of $\partial F$ to the other. Let $\bar{\varphi}_{a}$ be the surface obtained by removing $F$ and gluing the components of $\partial F$ together via $f$ (also making sure to preserve the marking); see the upper two surfaces in Figure 2. Let $\bar{\alpha}$ be the gluing curve in $\bar{\varphi}_{a}$. Since the vertical and horizontal foliations of $q_{a}$ match along $\bar{\alpha}$, the surface $\bar{\varphi}_{a}$ is naturally equipped with vertical and horizontal foliations $\bar{v}_{a}^{ \pm}$and quadratic differential $\bar{q}_{a}$, which is assumed to be scaled to have area one. Let $\left\{\bar{\varphi}_{t}\right\}$ be the Teichmüller geodesic corresponding to $\bar{q}_{a}$ and let $\bar{q}_{t}$ be the corresponding family of quadratic differentials. Then

$$
d_{\mathscr{T}(S)}\left(\overline{\mathscr{G}}_{a}, \overline{\mathscr{G}}_{b}\right)=b-a .
$$

Observe that the surface $\overline{\mathscr{G}}_{t}$ is obtained from $\mathscr{G}_{t}$ by cutting out the maximal flat annulus $F_{t}=F_{t}(\alpha)$. Thus, for each $t$, we have a natural map

$$
\varphi_{t}:\left(S, q_{t}\right) \backslash F_{t} \rightarrow\left(S, \bar{q}_{t}\right) \backslash \bar{\alpha}
$$

which fixes points but scales the metric. Hence, $K_{\bar{q}_{t}}(\alpha)=K_{t}(\alpha)>M$ on $[a, b]$ and therefore $\alpha$ is also extremely short in $\overline{\mathscr{G}}_{t}$ on $[a, b]$. Applying Theorem 2.4, we get

$$
b-a=d_{T_{(S)}}\left(\overline{\mathscr{G}}_{a}, \overline{\mathscr{G}}_{b}\right) \doteq \max \left\{d_{\mathscr{T}_{\left(S_{\alpha}\right)}}\left(\overline{\mathscr{G}}_{a}, \overline{\mathscr{G}}_{b}\right), d_{\mathbb{\mathbb { H } _ { \alpha } ^ { 2 }}}\left(\overline{\mathscr{G}}_{a}, \overline{\mathscr{G}}_{b}\right)\right\} .
$$

To prove the theorem, it will suffice to establish the following two bounds:

$$
d_{\mathbb{\mathbb { N } _ { \alpha } ^ { 2 }}}\left(\overline{\mathscr{G}}_{a}, \overline{\mathscr{G}}_{b}\right) \prec \log (b-a),
$$

and

$$
d_{\mathscr{T}\left(S_{\alpha}\right)}\left(\overline{\mathscr{G}}_{a}, \mathscr{G}_{a}\right) \prec 1, \quad d_{\mathscr{T}\left(S_{\alpha}\right)}\left(\overline{\mathscr{G}}_{b}, \mathscr{G}_{b}\right) \prec 1
$$


The theorem would then follow from (4-1), (4-2), (4-3) and the triangle inequality.

Proof of (4-2). We use the estimate of distance in $\mathbb{H}_{\alpha}^{2}$ from (2-1) in Section 2. Let $\sigma_{t}=\bar{G}_{t}$, let $\ell_{t}=l_{\sigma_{t}}(\alpha)$, and let $s_{t}$ be the Fenchel-Nielsen twist coordinate of $\alpha$ at $\sigma_{t}$. By (2-1) we have

$$
d_{\mathbb{\boxplus}_{\alpha}^{2}}\left(\sigma_{a}, \sigma_{b}\right) \succsim \frac{1}{2} \log \max \left\{\left|s_{a}-s_{b}\right|^{2} \ell_{a} \ell_{b}, \frac{\ell_{a}}{\ell_{b}}, \frac{\ell_{b}}{\ell_{a}}\right\} .
$$

We shall show that the contribution $\left|s_{a}-s_{b}\right|^{2} \ell_{a} \ell_{b}$ coming from the twist can be neglected. By Lemma 2.5, we have

$$
\left|s_{a}-s_{b}\right| \doteq\left|\mathrm{t} w_{\sigma_{a}}(\xi, \alpha)-\mathrm{t} w_{\sigma_{b}}(\xi, \alpha)\right|
$$

for any lamination $\xi$. By Proposition 2.7 with $\xi=v^{+}$(or $\xi=v^{-}$), we have

$$
\left|\mathrm{T} w_{\sigma_{a}}\left(v^{+}, \alpha\right)-\mathrm{T} w_{\bar{q}_{a}}\left(v^{+}, \alpha\right)\right| \prec \frac{1}{\ell_{a}}, \quad\left|\mathrm{~T} w_{\sigma_{b}}\left(v^{+}, \alpha\right)-\mathrm{T} w_{\bar{q}_{b}}\left(v^{+}, \alpha\right)\right| \prec \frac{1}{\ell_{b}} .
$$

In general, if a curve $\alpha$ is short on a surface $\sigma$ then, by considering the restriction to $F$, we can view $\mathrm{t} w_{q}(v, \alpha)$ as split into contributions coming from the flat and the expanding annuli around $\alpha$. It follows from the Gauss-Bonnet theorem that in an expanding annulus, two geodesics intersect at most once. Hence the contribution to $t w_{q}(\nu, \alpha)$ is essentially contained in $F(\alpha)$; for details see [Rafi 2007] and the proof of [Choi et al. 2006, Lemma 5.6].

In the present case, there is no flat annulus in $\bar{q}_{t}$ corresponding to $\alpha$. Hence the twistings $\mathrm{T} w_{\bar{q}_{a}}\left(v^{+}, \alpha\right)$ and $\mathrm{T} w_{\bar{q}_{b}}\left(v^{+}, \alpha\right)$ are bounded; in fact, they are at most two. Therefore, $\left|s_{a}-s_{b}\right|^{2} \ell_{a} \ell_{b} \prec 1$ and we get

$$
d_{\mathbb{H}_{\alpha}^{2}}\left(\sigma_{a}, \sigma_{b}\right) \doteq \frac{1}{2} \log \max \left\{\frac{\ell_{a}}{\ell_{b}}, \frac{\ell_{b}}{\ell_{a}}\right\} .
$$

Since $K_{\bar{q}_{a}}(\alpha)=K_{a}(\alpha)$ and $K_{\bar{q}_{b}}(\alpha)=K_{b}(\alpha)$, it follows from (3-3) that

$$
\frac{\ell_{a}}{\ell_{b}} \asymp \frac{\log K_{b}(\alpha)}{\log K_{a}(\alpha)} \prec \frac{2(b-a)+\log K_{a}(\alpha)}{\log K_{a}(\alpha)} \leq \frac{2(b-a)}{\log M}+1 .
$$

Similarly for $\ell_{b} / \ell_{a}$, we have the identical bound. Thus (4-2) is proved.

Proof of (4-3). This is a consequence of the following lemma due to Minsky.

Lemma 4.3 [Minsky 1992, Lemma 8.4]. Let X be a closed Riemann surface and $Y \subset X$ an incompressible subsurface. There exists a constant $m$ depending on the topology of $X$ only, such that if each component of $\partial Y$ bounds an annulus in $Y$ of modulus at least $m$, then for any nonperipheral simple closed curve $\zeta \subset Y$,

$$
\operatorname{Ext}_{\mathrm{Y}}(\zeta) \stackrel{*}{\curvearrowleft} \operatorname{Ext}_{\mathrm{X}}(\zeta)
$$


Here $\operatorname{Ext}_{Y}(\zeta)$ denotes the extremal length of a curve $\zeta$ on the surface $Y$. Note that although the lemma is stated for closed surfaces, the proof works for surfaces with punctures as well.

Continuing the proof of (4-3), for $t=a, b$ we claim that

$$
\operatorname{Ext}_{\varphi_{\mathrm{t}}}(\zeta) \stackrel{*}{\curvearrowleft} \operatorname{Ext}_{\overline{\varphi_{\mathrm{t}}}}(\zeta)
$$

for every nonperipheral simple closed curve $\zeta$ in $S \backslash \alpha$. Note that if the maximal flat annulus $F_{t}(\alpha)$ at $\left(S, q_{t}\right)$ has modulus bounded above by $m$, then there is a $k$ quasiconformal homeomorphism from $\left(S, q_{t}\right)$ to $\left(S, \bar{q}_{t}\right)$, where $k$ depends only on $m$. This automatically implies that

$$
d \mathscr{T}_{(S)}\left(\mathscr{G}_{t}, \overline{\mathscr{G}}_{t}\right) \prec 1 .
$$

Now suppose that $\operatorname{Mod} F_{t}(\alpha)>m$. In order to apply Lemma 4.3, we take the following intermediate step illustrated in Figure 2. As usual, $E_{t}(\alpha)$ is an expanding annulus of maximal modulus around $\alpha$. One component of $\partial F_{t}(\alpha)$ is the inner boundary $\partial_{0}$ of $E_{t}(\alpha)$. Let $\partial_{0}^{\prime}$ be the other component of $\partial F_{t}(\alpha)$ and let $A_{t}$ be the flat annulus contained in $F_{t}(\alpha)$ that shares $\partial_{0}^{\prime}$ as a boundary component and that has modulus $m$.

Let $\mathscr{L}_{t}$ be the surface which is obtained from $\mathscr{G}_{t}$ by cutting out $F_{t}(\alpha) \backslash A_{t}$ and regluing the boundary components together, shown as the lower surface in Figure 2. Each boundary component of $\mathscr{L}_{t} \backslash \partial_{0}$ has an annulus, namely $E_{t}(\alpha)$ and $A_{t}$, around it whose modulus is at least $m$. Therefore, we can apply Lemma 4.3 to $\mathscr{E}_{t} \backslash \partial_{0}$ as a subsurface of $\mathscr{G}_{t}$ and as a subsurface of $\mathscr{L}_{t}$ to obtain

$$
\operatorname{Ext}_{\varphi_{\mathrm{t}}}(\zeta) \stackrel{*}{\smile} \operatorname{Ext}_{\mathscr{L}_{\mathrm{t}} \backslash \partial_{0}}(\zeta) \stackrel{*}{`} \operatorname{Ext}_{\mathscr{L}_{\mathrm{t}}}(\zeta)
$$

Because the modulus of $A_{t}$ is bounded above by $m$, we have as above that $\mathscr{L}_{t}$ and $\bar{\varphi}_{t}$ are $k$-quasiconformal so that in particular,

$$
\operatorname{Ext}_{\mathscr{L}_{\mathrm{t}}}(\zeta) \stackrel{*}{\asymp} \operatorname{Ext}_{\bar{\varphi}_{\mathrm{t}}}(\zeta)
$$

This proves (4-4).

Thus it follows from the formulation of Teichmüller distance in [Kerckhoff 1980] and Minsky's product regions theorem that

$$
d_{\mathscr{T}\left(S_{\alpha}\right)}\left(\mathscr{G}_{t}, \bar{\varphi}_{t}\right) \succsim \frac{1}{2} \sup _{\zeta \in \mathscr{C}(S \backslash \alpha)} \log \frac{\operatorname{Ext}_{\varphi_{\mathrm{t}}}(\zeta)}{\operatorname{Ext}_{\bar{\varphi}_{\mathrm{t}}}(\zeta)} \prec 1,
$$

completing the proof of (4-3).

\section{The Main Theorem}

In this section we prove our main result, Theorem A. To estimate $d_{\mathcal{T}_{(S)}}\left(\mathscr{L}_{a}, \mathscr{L}_{b}\right)$ we will apply Minsky's product regions theorem and verify in turn upper and lower 
bounds on the distance. We start with a lemma which will be used to estimate $d_{\mathbb{W}_{\alpha}^{2}}\left(\mathscr{L}_{v}, \mathscr{L}_{w}\right)$, where $\alpha$ is a curve which is short along an interval $[v, w]$.

Recall from Proposition 2.1 that $I_{\alpha}=I_{\alpha}(\epsilon)$ is the maximal open interval around $t_{\alpha}$ such that $l \varphi_{t}(\alpha)<\epsilon$ for all $t \in I_{\alpha}$. It follows from Theorem 1.1 that if a curve is sufficiently short in $\mathscr{G}_{t}$, then it is, in the coarse sense, at least as short in $\mathscr{L}_{t}$. In particular, we may choose $\epsilon=\epsilon_{1}$ in Proposition 2.1 small enough that if $l_{\varphi_{t}}(\alpha)<\epsilon_{1}$, then $l_{\mathscr{L}_{t}}(\alpha)<\epsilon_{0}$.

Lemma 5.1. Let $[v, w] \subset I_{\alpha}\left(\epsilon_{1}\right)$.

(i) If $D_{t}(\alpha) \geq \sqrt{K_{t}(\alpha)}$ for all $t \in[v, w]$, then

$$
d_{\mathbb{H}_{\alpha}^{2}}\left(\mathscr{L}_{v}, \mathscr{L}_{w}\right) \doteq w-v .
$$

(ii) If $\sqrt{K_{t}(\alpha)} \geq D_{t}(\alpha)$ for all $t \in[v, w]$, then

$$
d_{\mathbb{\mathbb { W } _ { \alpha } ^ { 2 }}}\left(\mathscr{L}_{v}, \mathscr{L}_{w}\right) \stackrel{ \pm}{\prec} \frac{w-v}{2} .
$$

Proof. The proof rests on the formula (2-1) from Section 2 and a careful comparison of rates of change of lengths and twists. Let $\ell_{t}=l_{\mathscr{L}_{t}}(\alpha)$, and let $s_{t}$ be the FenchelNielsen twist of $\alpha$ at $\mathscr{L}_{t}$. As in (2-1) we have

$$
d_{\mathbb{N}_{\alpha}^{2}}\left(\mathscr{L}_{v}, \mathscr{L}_{w}\right) \succsim \frac{1}{2} \log \max \left\{\left|s_{v}-s_{w}\right|^{2} \ell_{v} \ell_{w}, \frac{\ell_{v}}{\ell_{w}}, \frac{\ell_{w}}{\ell_{v}}\right\} .
$$

By Lemma 2.5, we have

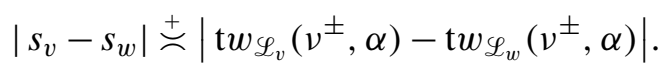

First suppose $t_{\alpha} \leq v<w$. Then by Theorem 2.9,

$$
\left|s_{v}-s_{w}\right|^{2} \ell_{v} \ell_{w} \stackrel{*}{\prec}\left(\frac{1}{\ell_{v}}+\frac{1}{\ell_{w}}\right)^{2} \ell_{v} \ell_{w} \stackrel{*}{\smile} \max \left\{\frac{\ell_{v}}{\ell_{w}}, \frac{\ell_{w}}{\ell_{v}}\right\} .
$$

Therefore,

$$
d_{\mathbb{\boxplus}_{\alpha}^{2}}\left(\mathscr{L}_{v}, \mathscr{L}_{w}\right) \succsim \frac{1}{2} \log \max \left\{\frac{\ell_{v}}{\ell_{w}}, \frac{\ell_{w}}{\ell_{v}}\right\} .
$$

If $D_{t}(\alpha) \geq \sqrt{K_{t}(\alpha)}$ for all $t \in[v, w]$, so that $1 / l_{\mathscr{L}_{t}}(\alpha) \stackrel{*}{`} D_{t}(\alpha)$ on $[v, w]$, then

$$
\max \left\{\frac{\ell_{v}}{\ell_{w}}, \frac{\ell_{w}}{\ell_{v}}\right\} \stackrel{*}{\star} e^{2(w-v)} .
$$

If $\sqrt{K_{t}(\alpha)} \geq D_{t}(\alpha)$ for all $t \in[v, w]$, so that $1 / l_{\mathscr{L}_{t}}(\alpha) \stackrel{*}{\asymp} \sqrt{K_{t}(\alpha)}$ on $[v, w]$, then by (3-3) and Lemma 3.1, we get

$$
\sqrt{K_{w}(\alpha)} \stackrel{*}{\prec} \sqrt{K_{v}(\alpha)} \stackrel{*}{\prec} e^{w-v} \sqrt{K_{w}(\alpha)},
$$


from which it follows that

$$
\max \left\{\frac{\ell_{v}}{\ell_{w}}, \frac{\ell_{w}}{\ell_{v}}\right\} \stackrel{*}{\prec} e^{w-v}
$$

and the lemma is proved in this case. The case where $v<w \leq t_{\alpha}$ can be handled similarly.

Now, suppose $v<t_{\alpha}<w$ and for convenience, translate so that $t_{\alpha}=0$. If $\sqrt{K_{t}(\alpha)} \geq D_{t}(\alpha)$ for all $t \in[v, w]$, the result follows from the triangle inequality

$$
d_{\mathbb{\mathbb { H } _ { \alpha } ^ { 2 }}}\left(\mathscr{L}_{v}, \mathscr{L}_{w}\right) \leq d_{\mathbb{\boxplus}_{\alpha}^{2}}\left(\mathscr{L}_{v}, \mathscr{L}_{0}\right)+d_{\mathbb{\mathbb { W } _ { \alpha } ^ { 2 }}}\left(\mathscr{L}_{0}, \mathscr{L}_{w}\right)
$$

and is already proved above.

The interesting case is that in which $D_{t}(\alpha) \geq \sqrt{K_{t}(\alpha)}$ for all $t \in[v, w]$, in which case $l_{\mathscr{L}_{t}}(\alpha)$ decreases on $[v, 0]$ but then increases again on $[0, w]$. This means that

$$
\max \left\{\frac{\ell_{v}}{\ell_{w}}, \frac{\ell_{w}}{\ell_{v}}\right\} \stackrel{*}{\approx} e^{2|v+w|}
$$

and consequently the term

$$
\frac{1}{2} \log \max \left\{\frac{\ell_{v}}{\ell_{w}}, \frac{\ell_{w}}{\ell_{v}}\right\}
$$

does not reflect the total distance $w-v$. Instead, we have to look more carefully at the term $\left|s_{v}-s_{w}\right|^{2} \ell_{v} \ell_{w}$.

We have

$$
\left|s_{v}-s_{w}\right| \ell_{v} \succsim\left|\mathrm{t} w \mathscr{L}_{v}\left(v^{-}, \alpha\right)-\mathrm{t} w \mathscr{L}_{w}\left(v^{-}, \alpha\right)\right| \ell_{v} \doteq \mathrm{T} w \mathscr{L}_{w}\left(v^{-}, \alpha\right) \ell_{v}
$$

where the second equality follows from Theorem 2.9. Similarly,

$$
\left|s_{v}-s_{w}\right| \ell_{w} \succsim\left|\mathrm{t} w_{\mathscr{L}_{v}}\left(v^{+}, \alpha\right)-\mathrm{t} w \mathscr{L}_{w}\left(v^{+}, \alpha\right)\right| \ell_{w} \succsim \mathrm{T} w \mathscr{L}_{v}\left(v^{+}, \alpha\right) \ell_{w} .
$$

On the other hand, writing $d_{\alpha}=d_{\alpha}\left(v^{+}, v^{-}\right)$for the relative twist as defined in Section 2, we get

$$
\begin{aligned}
& d_{\alpha} \ell_{v} \doteq\left|\mathrm{t} w_{\mathscr{L}_{v}}\left(v^{-}, \alpha\right)-\mathrm{t} w_{\mathscr{L}_{v}}\left(v^{+}, \alpha\right)\right| \ell_{v} \pm \mathrm{T} w_{\mathscr{L}_{v}}\left(v^{+}, \alpha\right) \ell_{v}, \\
& d_{\alpha} \ell_{w} \doteq\left|\mathrm{t} w_{\mathscr{L}_{w}}\left(v^{-}, \alpha\right)-\mathrm{t} w_{\mathscr{L}_{w}}\left(v^{+}, \alpha\right)\right| \ell_{w} \doteq \mathrm{T} w_{\mathscr{L}_{w}}\left(v^{-}, \alpha\right) \ell_{w},
\end{aligned}
$$

where we again made two applications of Theorem 2.9.

Also note that by definition, $D_{0}(\alpha)=d_{\alpha}$ so by Theorem 1.1 we have $1 / \ell_{0} \stackrel{*}{=} d_{\alpha}$. Thus

$$
\left|s_{v}-s_{w}\right|^{2} \ell_{v} \ell_{w} \stackrel{*}{`} d_{\alpha}^{2} \ell_{v} \ell_{w} \stackrel{*}{\longleftarrow} \frac{\ell_{v}}{\ell_{0}} \frac{\ell_{w}}{\ell_{0}}=e^{2(w-v)} .
$$

It follows by (5-1) that

$$
d_{\mathbb{H}_{\alpha}^{2}}\left(\mathscr{L}_{v}, \mathscr{L}_{w}\right) \succsim w-v .
$$


Before proving our main theorem, we also establish the following rather technical lemma, which quantifies more precisely the schematic graphs in Figure 1.

Lemma 5.2. Let $M$ be chosen as in Theorem 4.1. Then there exists $\epsilon>0$, depending only on the topology of $S$, such that for any $a, b$ with $[a, b] \subset I_{\alpha}(\epsilon)$, one of the following alternatives holds:

(i) $K_{t}(\alpha)>M$ on $[a, b]$;

(ii) item (i) fails, $D_{t}(\alpha) \geq \sqrt{K_{t}(\alpha)}$ on a subinterval of the form $[a, u]$ and

$$
\sqrt{K_{b}(\alpha)} \stackrel{*}{\prec} e^{u-a} ;
$$

(iii) item (i) fails, $D_{t}(\alpha) \geq \sqrt{K_{t}(\alpha)}$ on a subinterval of the form $[u, b]$ and

$$
\sqrt{K_{a}(\alpha)} \stackrel{*}{\prec} e^{b-u} .
$$

Proof. By Theorem 1.1, we can choose $\epsilon>0$ small enough that if $t \in I_{\alpha}(\epsilon)$ and if $\sqrt{K_{t}(\alpha)} \geq D_{t}(\alpha)$ then $K_{t}(\alpha)>M$. Thus if (i) fails, we must have $D_{w}(\alpha)>$ $\sqrt{K_{w}(\alpha)}$ for some $w \in(a, b)$.

Suppose first that $D_{t}(\alpha)>\sqrt{K_{t}(\alpha)}$ on $[a, b]$, and that (i) fails, so that there is some $c \in[a, b]$ where $K_{c}(\alpha) \leq M$. To check (ii) holds, we only have to verify its final statement. Since $M$ is fixed, it follows from (3-3) that

$$
\sqrt{K_{b}(\alpha)} \stackrel{*}{\prec} e^{b-c} \sqrt{K_{c}(\alpha)} \stackrel{*}{\prec} e^{b-a} .
$$

(By the same argument, (iii) also holds in this case.)

Now suppose that $D_{u}(\alpha)=\sqrt{K_{u}(\alpha)}$ for some $u \in(a, b)$. We claim that if $t_{\alpha} \notin[a, b]$ then (i) holds. Suppose for definiteness that $t_{\alpha}<a$. By Lemma 3.1 we have $K_{t}(\alpha) \stackrel{*}{\succ} K_{u}(\alpha)$ on $[a, u]$, and by Lemma 3.2 we have $\sqrt{K_{t}(\alpha)} \stackrel{*}{\succ}^{\circ}(\alpha)$ on $[u, b]$. Hence $1 / l_{\mathscr{L}_{t}}(\alpha) \asymp \sqrt{K_{t}(\alpha)}$ on $[u, b]$. Therefore, reducing $\epsilon>0$ if necessary, we can again ensure $K_{t}(\alpha)>M$ on $[a, b]$ and (i) holds as claimed.

Suppose now that $D_{u}(\alpha)=\sqrt{K_{u}(\alpha)}$ for some $u \in[a, b]$ and that $t_{\alpha} \in[a, b]$, say for definiteness that $t_{\alpha}<u$. If there is another point $u^{\prime} \in\left[a, t_{\alpha}\right]$ such that $D_{u^{\prime}}(\alpha)=\sqrt{K_{u^{\prime}}(\alpha)}$, then again with a suitable adjustment of $\epsilon$ we have $K_{t}(\alpha)>M$ on $[a, b]$ (see Figure 1) and we are in case (i). If there is no such point $u^{\prime}$, then $D_{t}(\alpha) \geq \sqrt{K_{t}(\alpha)}$ on $[a, u]$. Assuming that in addition (i) fails, there is a point $c \in[a, b]$ where $K_{c}(\alpha) \leq M$. By Lemma 3.2 we have $1 / l_{\mathscr{L}_{t}}(\alpha) \stackrel{*}{\curvearrowleft} \sqrt{K_{t}(\alpha)}$ on $[u, b]$. Assuming $\epsilon$ is sufficiently small, we deduce that $c \in[a, u]$. Then by Lemma 3.1 we have

$$
\sqrt{K_{c}(\alpha)} \stackrel{*}{\succ} e^{-\left|t_{\alpha}-c\right|} \sqrt{K_{t_{\alpha}}(\alpha)} \stackrel{*}{\succ} e^{-\left|t_{\alpha}-c\right|} \sqrt{K_{b}(\alpha)} \geq e^{-(u-a)} \sqrt{K_{b}(\alpha)}
$$

and we are in case (ii). The case where $t_{\alpha}>u$ is handled similarly and results in (iii). 
Proof of Theorem A. As noted in Section 1, we prove the theorem by obtaining separate upper and lower bounds for $d_{T_{(S)}}\left(\mathscr{L}_{a}, \mathscr{L}_{b}\right)$. The upper bound is relatively straightforward but the lower bound requires an inductive procedure based on Lemma 5.2.

In order to compare two surfaces $\mathscr{L}_{a}, \mathscr{L}_{b}$ at the ends of a long interval $[a, b] \subset \mathbb{R}$, it is convenient to consider separately the curves which are short at $a$ but not at $b$, those which are short at $b$ but not at $a$, and those which are short at both. More precisely, choose $\epsilon$ to satisfy Lemma 5.2 and then choose $\epsilon^{\prime} \leq \epsilon$ as in Proposition 2.1 so that if $l \varphi_{\varphi_{t}}(\alpha)<\epsilon^{\prime}$ then $t \in I_{\alpha}(\epsilon)$. In particular, if $l \varphi_{a}(\alpha)<\epsilon^{\prime}$ and $l \varphi_{b}(\alpha)<\epsilon^{\prime}$, then since $I_{\alpha}(\epsilon)$ is connected, $[a, b] \subset I_{\alpha}(\epsilon)$. Now define subsets $\Gamma_{a}, \Gamma_{b}$ and $\Gamma$ of the curves of length less than $\epsilon^{\prime}$ in either $\mathscr{G}_{a}$ or $\mathscr{G}_{b}$ as follows:

$$
\begin{aligned}
& \Gamma_{a}=\left\{\alpha \in \mathscr{C}(S): l \varphi_{a}(\alpha)<\epsilon^{\prime}, l \varphi_{\varphi_{b}}(\alpha) \geq \epsilon^{\prime}\right\}, \\
& \Gamma_{b}=\left\{\alpha \in \mathscr{C}(S): l \varphi_{b}(\alpha)<\epsilon^{\prime}, l \varphi_{a}(\alpha) \geq \epsilon^{\prime}\right\}, \\
& \Gamma=\left\{\alpha \in \mathscr{C}(S): l \varphi_{\varphi_{t}}(\alpha)<\epsilon^{\prime}, \text { for } t=a, b\right\} .
\end{aligned}
$$

We begin by establishing some preliminary estimates on distances in the Teichmüller spaces of the subsurfaces obtained by cutting along these curves. By Minsky's product regions theorem,

$$
\begin{aligned}
& d_{\mathscr{T}_{\left(S_{\Gamma}\right)}}\left(\mathscr{L}_{a}, \mathscr{G}_{a}\right) \doteq \max _{\alpha \in \Gamma_{a}}\left\{d_{\mathscr{T}_{\left(S_{\Gamma \cup \Gamma_{a}}\right)}}\left(\mathscr{L}_{a}, \mathscr{G}_{a}\right), d_{\mathbb{\boxplus}_{\alpha}^{2}}\left(\mathscr{L}_{a}, \mathscr{G}_{a}\right)\right\}, \\
& d_{\mathcal{T}_{\left(S_{\Gamma}\right)}}\left(\mathscr{L}_{b}, \mathscr{G}_{b}\right) \succsim \max _{\alpha \in \Gamma_{b}}\left\{d_{\left.\mathscr{T}_{\left(S_{\Gamma \cup \Gamma_{b}}\right.}\right)}\left(\mathscr{L}_{b}, \mathscr{G}_{b}\right), d_{\mathbb{H}_{\alpha}^{2}}\left(\mathscr{L}_{b}, \mathscr{G}_{b}\right)\right\} .
\end{aligned}
$$

Now on the one hand, by Theorem 1.2, the thick parts of $\mathscr{G}_{a}$ and $\mathscr{L}_{a}$ are bounded distance from one another, as are the those of $\mathscr{G}_{b}$ and $\mathscr{L}_{b}$. Therefore,

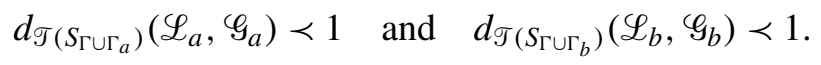

On the other hand, because the twisting is bounded as in Theorems 2.8 and 2.9, we have

$$
\begin{aligned}
& d_{\mathbb{W}_{\alpha}^{2}}\left(\mathscr{L}_{a}, \mathscr{G}_{a}\right) \stackrel{ \pm}{2} \log \frac{\lg _{g_{a}}(\alpha)}{l_{\mathscr{L}_{a}}(\alpha)}<\frac{1}{2} \log \frac{1}{l_{\mathscr{L}_{a}}(\alpha)} \quad \text { for } \alpha \in \Gamma_{a}, \\
& d_{\mathbb{N}_{\alpha}^{2}}\left(\mathscr{L}_{b}, \mathscr{G}_{b}\right) \stackrel{ \pm}{2} \log \frac{l \mathscr{\varphi}_{b}(\alpha)}{l_{\mathscr{L}_{b}}(\alpha)}<\frac{1}{2} \log \frac{1}{l_{\mathscr{L}_{b}}(\alpha)} \quad \text { for } \alpha \in \Gamma_{b} .
\end{aligned}
$$

Thus it follows that

$$
\begin{aligned}
& d_{\mathscr{T}_{\left(S_{\Gamma}\right)}}\left(\mathscr{L}_{a}, \mathscr{G}_{a}\right) \stackrel{+}{\prec} \max _{\alpha \in \Gamma_{a}}\left\{\log \frac{1}{l_{\mathscr{L}_{a}}(\alpha)}\right\}, \\
& d_{\mathscr{T}_{\left(S_{\Gamma}\right)}}\left(\mathscr{L}_{b}, \mathscr{G}_{b}\right) \stackrel{1}{\prec} \max _{\alpha \in \Gamma_{b}}\left\{\log \frac{1}{l_{\mathscr{L}_{b}}(\alpha)}\right\} .
\end{aligned}
$$


We now turn to bounding the distance $d_{\mathcal{T}(S)}\left(\mathscr{L}_{a}, \mathscr{L}_{b}\right)$. By Minsky's product regions theorem,

$$
d_{\mathscr{T}(S)}\left(\mathscr{L}_{a}, \mathscr{L}_{b}\right) \doteq \max _{\alpha \in \Gamma}\left\{d_{\mathscr{T}\left(S_{\Gamma}\right)}\left(\mathscr{L}_{a}, \mathscr{L}_{b}\right), d_{\mathbb{W}_{\alpha}^{2}}\left(\mathscr{L}_{a}, \mathscr{L}_{b}\right)\right\} .
$$

Upper bound. We prove the upper bound $d_{T_{(S)}}\left(\mathscr{L}_{a}, \mathscr{L}_{b}\right) \stackrel{\star}{\prec}(b-a)$ by bounding the terms on the right hand side of (5-3).

By Lemma 5.1 we have $d_{\mathbb{N}_{\alpha}^{2}}\left(\mathscr{L}_{a}, \mathscr{L}_{b}\right) \stackrel{\star}{\prec} b-a$ for each $\alpha \in \Gamma$. We provide an upper bound for $d_{\mathscr{T}_{\left(S_{\Gamma}\right)}}\left(\mathscr{L}_{a}, \mathscr{L}_{b}\right)$ using the triangle inequality

$$
d \mathscr{T}_{\left(S_{\Gamma}\right)}\left(\mathscr{L}_{a}, \mathscr{L}_{b}\right) \leq d_{\mathcal{T}_{\left(S_{\Gamma}\right)}}\left(\mathscr{L}_{a}, \mathscr{G}_{a}\right)+d_{\mathcal{T}_{\left(S_{\Gamma}\right)}}\left(\mathscr{G}_{a}, \mathscr{G}_{b}\right)+d_{\mathcal{T}_{\left(S_{\Gamma}\right)}}\left(\mathscr{L}_{b}, \mathscr{G}_{b}\right) .
$$

To bound the first and last terms of the right hand side, we will use (5-2) and the fact that the length $l_{\mathscr{L}_{t}}(\alpha)$ of a curve increases at rate at most $e^{2 t}$. More precisely, notice that if $\alpha \in \Gamma_{a}$ then $I_{\alpha} \cap[a, b]=[a, c)$ for some $c \leq b$. By definition of $I_{\alpha}$, we have $l \varphi_{c}(\alpha)=\epsilon$. Then it follows from Theorem 1.1 that $l_{\mathscr{L}_{c}}(\alpha)$ is bounded below by a uniform constant that depends only on $\epsilon$. Therefore, by the observation following (3-3), we have

$$
\log \frac{1}{l_{\mathscr{L}_{a}}(\alpha)} \stackrel{+}{\gtrless} \log \frac{l_{\mathscr{L}_{c}}(\alpha)}{l_{\mathscr{L}_{a}}(\alpha)} \gtrless 2(b-a) .
$$

Similarly, if $\alpha \in \Gamma_{b}$, then

$$
\log \frac{1}{l_{\mathscr{L}_{b}}(\alpha)} \stackrel{+}{\prec} 2(b-a) .
$$

Therefore, from (5-2) it follows that

$$
d_{\mathcal{T}_{\left(S_{\Gamma}\right)}}\left(\mathscr{L}_{a}, \mathscr{G}_{a}\right) \stackrel{\star}{\prec} b-a \text { and } d_{\mathscr{T}_{\left(S_{\Gamma}\right)}}\left(\mathscr{L}_{b}, \mathscr{G}_{b}\right) \stackrel{+}{\prec} b-a \text {. }
$$

The second term in (5-4) is bounded by Minsky's product regions theorem:

$$
d_{T_{\left(S_{\Gamma}\right)}}\left(\varphi_{a}, \mathscr{G}_{b}\right) \stackrel{ \pm}{\prec} d_{T_{(S)}}\left(\mathscr{G}_{a}, \mathscr{G}_{b}\right)=b-a .
$$

This finishes the proof of the upper bound.

Lower bound. We prove the lower bound $d_{T_{(S)}}\left(\mathscr{L}_{a}, \mathscr{L}_{b}\right) \stackrel{+}{\succ}(b-a) / 4$ by showing that at least one of the terms in the right hand side of (5-3) is bounded below by $(b-a) / 4$.

We begin by reducing the problem to a consideration of the curves in $\Gamma$ only. It follows from a theorem of Wolpert [1979] that for every $\gamma \in \mathscr{C}(S)$,

$$
d_{\mathscr{T}_{(S)}}\left(\mathscr{L}_{a}, \mathscr{L}_{b}\right) \geq \frac{1}{2}\left|\log \frac{l_{\mathscr{L}_{b}}(\gamma)}{l_{\mathscr{L}_{a}}(\gamma)}\right| .
$$


It follows as before from Theorem 1.1 that if $l \varphi_{\varphi_{t}}(\alpha) \geq \epsilon^{\prime}$, then the length $l_{\mathscr{L}_{t}}(\alpha)$ is uniformly bounded below. Therefore, we have

$$
\begin{aligned}
& \left|\log \frac{l_{\mathscr{L}_{b}}(\alpha)}{l_{\mathscr{L}_{a}}(\alpha)}\right| \stackrel{+}{\succ} \log \frac{1}{l_{\mathscr{L}_{a}}(\alpha)} \quad \text { for } \alpha \in \Gamma_{a}, \\
& \left|\log \frac{l_{\mathscr{L}_{a}}(\alpha)}{l_{\mathscr{L}_{b}}(\alpha)}\right| \stackrel{+}{\succ} \log \frac{1}{l_{\mathscr{L}_{b}}(\alpha)} \quad \text { for } \alpha \in \Gamma_{b} .
\end{aligned}
$$

It follows from the triangle inequality that if either

$$
\max _{\alpha \in \Gamma_{a}}\left\{\log \frac{1}{l_{\mathscr{L}_{a}}(\alpha)}\right\} \geq \frac{b-a}{2} \text { or } \max _{\alpha \in \Gamma_{b}}\left\{\log \frac{1}{l_{\mathscr{L}_{b}}(\alpha)}\right\} \geq \frac{b-a}{2},
$$

the lower bound is proved.

Thus we may assume that

$$
\max _{\alpha \in \Gamma_{a}}\left\{\log \frac{1}{l_{\mathscr{L}_{a}}(\alpha)}\right\} \leq \frac{b-a}{2} \text { and } \max _{\alpha \in \Gamma_{b}}\left\{\log \frac{1}{l_{\mathscr{L}_{b}}(\alpha)}\right\} \leq \frac{b-a}{2},
$$

bringing us to the key part of the proof. From Minsky's product region theorem, we have

$$
b-a=d_{\mathscr{T}_{(S)}}\left(\mathscr{G}_{a}, \mathscr{G}_{b}\right) \doteq \max _{\alpha \in \Gamma}\left\{d_{\mathcal{T}_{\left(S_{\Gamma}\right)}}\left(\mathscr{G}_{a}, \mathscr{G}_{b}\right), d_{\mathbb{H}_{\alpha}^{2}}\left(\mathscr{G}_{a}, \mathscr{G}_{b}\right)\right\} .
$$

We claim that either

$$
d \mathscr{T}_{\left(S_{\Gamma}\right)}\left(\mathscr{G}_{a}, \mathscr{G}_{b}\right) \succsim b-a,
$$

or there is some $\alpha \in \Gamma$ such that

$$
d_{\mathbb{\mathbb { N } _ { \alpha } ^ { 2 }}}\left(\mathscr{G}_{a}, \mathscr{G}_{b}\right) \succsim b-a
$$

and such that Lemma 5.2 (ii) or (iii) holds. After proving the claim, we will show that either alternative implies the required bound on $d_{\mathcal{T}(S)}\left(\mathscr{L}_{a}, \mathscr{L}_{b}\right)$. We are going to use an inductive argument for which it is important to note that we can choose the additive constant in Minsky's product regions theorem to be fixed for all surfaces obtained from $S$ by cutting out any subset of curves in $\Gamma$.

If the maximum in (5-6) is realized by $d_{\mathcal{T}_{\left(S_{\Gamma}\right)}}\left(\mathscr{G}_{a}, \mathscr{G}_{b}\right)$, then obviously

$$
d \mathscr{T}_{\left(S_{\Gamma}\right)}\left(\mathscr{G}_{a}, \mathscr{G}_{b}\right) \doteq b-a .
$$

If the maximum in (5-6) is realized by $d_{\mathbb{W}_{\gamma}^{2}}\left(\mathscr{G}_{a}, \mathscr{G}_{b}\right)$ for some $\gamma \in \Gamma$, consider the alternatives for $\gamma$ in Lemma 5.2. If (i) holds, then by Theorem 4.1 we have

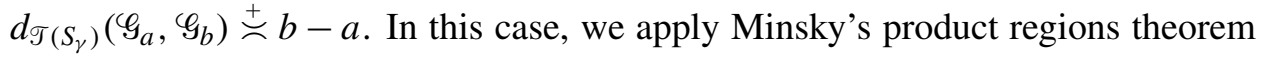
to $S_{\gamma}$, giving

$$
b-a \doteq d_{\mathscr{T}\left(S_{\gamma}\right)}\left(\mathscr{G}_{a}, \mathscr{G}_{b}\right) \doteq \max _{\delta \in \Gamma \backslash \gamma}\left\{d_{\mathscr{T}_{\left(S_{\Gamma}\right)}}\left(\mathscr{G}_{a}, \mathscr{G}_{b}\right), d_{\mathbb{W}_{\delta}^{2}}\left(\mathscr{G}_{a}, \mathscr{G}_{b}\right)\right\} .
$$


Now repeat the same argument; if the maximum in (5-7) is realized by $d_{\mathbb{W}}\left(\mathscr{G}_{a}, \mathscr{G}_{b}\right)$ for some $\delta \in \Gamma \backslash \gamma$ that satisfies Lemma 5.2 (i), then apply the product regions theorem to $S_{\{\gamma, \delta\}}$. Eventually, up to a finite number of changes to the additive constants, either there must be some $\alpha \in \Gamma$ for which $d_{\mathbb{W}_{\alpha}^{2}}\left(\varphi_{a}, \mathscr{G}_{b}\right) \succsim b-a$ such that Lemma 5.2 (i) does not hold, or it must be that $d_{\mathcal{T}_{\left(S_{\Gamma}\right)}}\left(\mathscr{G}_{a}, \mathscr{G}_{b}\right) \succsim b-a$. The claim follows.

Now we show that either alternative implies the required bound. If

$$
d_{\mathscr{T}\left(S_{\Gamma}\right)}\left(\mathscr{G}_{a}, \mathscr{G}_{b}\right) \succsim b-a,
$$

then the triangle inequality, (5-2), and the assumption (5-5) give

$$
\begin{aligned}
& d_{\mathscr{T}\left(S_{\Gamma}\right)}\left(\mathscr{L}_{a}, \mathscr{L}_{b}\right) \stackrel{+}{\succ} d_{\mathscr{T}\left(S_{\Gamma}\right)}\left(\mathscr{G}_{a}, \mathscr{G}_{b}\right)-d_{T_{\left(S_{\Gamma}\right)}}\left(\mathscr{L}_{a}, \mathscr{G}_{a}\right)-d_{\mathscr{T}\left(S_{\Gamma}\right)}\left(\mathscr{L}_{b}, \mathscr{G}_{b}\right) \\
& \stackrel{+}{\succ} d_{\mathcal{T}_{\left(S_{\Gamma}\right)}}\left(\mathscr{G}_{a}, \mathscr{G}_{b}\right)-\frac{1}{2} \max _{\alpha \in \Gamma_{a}}\left\{\log \frac{1}{l_{\mathscr{L}_{a}}(\alpha)}\right\}-\frac{1}{2} \max _{\alpha \in \Gamma_{b}}\left\{\log \frac{1}{l_{\mathscr{L}_{b}}(\alpha)}\right\} \\
& \pm \frac{b-a}{2} \text {. }
\end{aligned}
$$

Now assume the alternative that there is some $\alpha \in \Gamma$ such that $d_{\mathbb{~} \uplus_{\alpha}^{2}}\left(\mathscr{G}_{a}, \mathscr{G}_{b}\right) \succsim$ $b-a$ and such that Lemma 5.2 (ii) or (iii) holds. Assume (ii) holds: we have that $D_{t}(\alpha) \geq \sqrt{K_{t}(\alpha)}$ on an interval $[a, u]$ and consider the following two cases depending on the length of $[a, u]$. (Case (iii) can be handled similarly.)

If $u-a \geq(b-a) / 2$, then the triangle inequality and Lemma 5.1 give

$$
\begin{aligned}
d_{\mathbb{\boxplus}_{\alpha}^{2}}\left(\mathscr{L}_{a}, \mathscr{L}_{b}\right) & \geq d_{\mathbb{\boxplus}_{\alpha}^{2}}\left(\mathscr{L}_{a}, \mathscr{L}_{u}\right)-d_{\mathbb{\boxplus}_{\alpha}^{2}}\left(\mathscr{L}_{u}, \mathscr{L}_{b}\right) \\
& +\frac{b}{\succ}(u-a)-\frac{b-u}{2} \\
& \geq \frac{b-a}{4} .
\end{aligned}
$$

(Strictly speaking, it may be that $\sqrt{K_{t}(\alpha)}<D_{t}(\alpha)$ for some values of $t \in[u, b]$. However, Lemma 3.2 implies that $1 / l_{\mathscr{L}_{t}}(\alpha) \stackrel{*}{\curvearrowleft} \sqrt{K_{t}(\alpha)}$ on $[u, b]$, and this is sufficient to guarantee that $d_{\mathbb{U}_{\alpha}^{2}}\left(\mathscr{L}_{u}, \mathscr{L}_{b}\right) \stackrel{\gtrless}{\gtrless}(b-u) / 2$; see the proof of Lemma 5.1.)

If $u-a<(b-a) / 2$, then consider the triangle inequality

$$
d_{\mathbb{\mathbb { W } _ { \alpha } ^ { 2 }}}\left(\mathscr{L}_{a}, \mathscr{L}_{b}\right) \geq d_{\mathbb{W}_{\alpha}^{2}}\left(\mathscr{G}_{a}, \mathscr{G}_{b}\right)-d_{\mathbb{H}_{\alpha}^{2}}\left(\mathscr{G}_{a}, \mathscr{L}_{a}\right)-d_{\mathbb{W}_{\alpha}^{2}}\left(\mathscr{G}_{b}, \mathscr{L}_{b}\right)
$$

Similarly to our previous argument, since the twisting is bounded as in Theorems 2.8 and 2.9, we have

$$
d_{\mathbb{\boxplus}_{\alpha}^{2}}\left(\mathscr{G}_{a}, \mathscr{L}_{a}\right) \stackrel{+}{2} \log \frac{l \mathscr{g}_{a}(\alpha)}{l_{\mathscr{L}_{a}}(\alpha)} \quad \text { and } \quad d_{\mathbb{\boxplus}_{\alpha}^{2}}\left(\mathscr{G}_{b}, \mathscr{L}_{b}\right) \stackrel{+}{2} \log \frac{l \mathscr{\varphi}_{b}(\alpha)}{l_{\mathscr{L}_{b}}(\alpha)} \text {. }
$$


Since $D_{a}(\alpha) \geq \sqrt{K_{a}(\alpha)}$ it follows from Theorem 1.1 that

$$
\log \frac{l_{\varphi_{a}}(\alpha)}{l_{\mathscr{L}_{a}}(\alpha)} \stackrel{*}{\approx} 1 \text {. }
$$

Since Lemma 5.2 (ii) holds, it follows from the assumption $u-a<(b-a) / 2$ that

$$
\log \frac{l_{\varphi_{b}}(\alpha)}{l_{\mathscr{L}_{b}}(\alpha)}<\log \frac{1}{l_{\mathscr{L}_{b}}(\alpha)} \stackrel{+}{\gtrless} u-a<\frac{b-a}{2} .
$$

Thus, in this case we have

$$
d_{\mathbb{\mathbb { N } _ { \alpha } ^ { 2 }}}\left(\mathscr{L}_{a}, \mathscr{L}_{b}\right) \stackrel{+}{\succ} \frac{3}{4}(b-a) .
$$

This concludes the proof.

\section{Acknowledgment}

We would like to thank the referee for helpful comments.

\section{References}

[Choi and Rafi 2007] Y.-E. Choi and K. Rafi, "Comparison between Teichmüller and Lipschitz metrics", J. Lond. Math. Soc. (2) 76:3 (2007), 739-756. MR 2377122 Zbl 1132.30024

[Choi et al. 2006] Y. Choi, K. Rafi, and C. Series, "Lines of minima and Teichmüller geodesics", preprint, 2006. To appear in Geom. Funct. Anal. arXiv math.GT/0605135

[Gardiner and Masur 1991] F. P. Gardiner and H. Masur, "Extremal length geometry of Teichmüller space", Complex Variables Theory Appl. 16:2-3 (1991), 209-237. MR 92f:32034 Zbl 0702.32019

[Kerckhoff 1980] S. P. Kerckhoff, "The asymptotic geometry of Teichmüller space”, Topology 19:1 (1980), 23-41. MR 81f:32029 Zbl 0439.30012

[Kerckhoff 1992] S. P. Kerckhoff, "Lines of minima in Teichmüller space", Duke Math. J. 65:2 (1992), 187-213. MR 93b:32027 Zbl 0771.30043

[Levitt 1983] G. Levitt, "Foliations and laminations on hyperbolic surfaces", Topology 22:2 (1983), 119-135. MR 84h:57015 Zbl 0522.57027

[Masur and Minsky 2000] H. A. Masur and Y. N. Minsky, "Geometry of the complex of curves. II. Hierarchical structure", Geom. Funct. Anal. 10:4 (2000), 902-974. MR 2001k:57020 Zbl 0972. 32011

[Minsky 1992] Y. N. Minsky, "Harmonic maps, length, and energy in Teichmüller space", J. Differential Geom. 35:1 (1992), 151-217. MR 93e:58041 Zbl 0763.53042

[Minsky 1996] Y. N. Minsky, "Extremal length estimates and product regions in Teichmüller space", Duke Math. J. 83:2 (1996), 249-286. MR 97b:32019 Zbl 0861.32015

[Rafi 2005] K. Rafi, "A characterization of short curves of a Teichmüller geodesic”, Geom. Topol. 9 (2005), 179-202. MR 2005i:30072 Zbl 1082.30037

[Rafi 2007] K. Rafi, "A combinatorial model for the Teichmüller metric”, Geom. Funct. Anal. 17:3 (2007), 936-959. MR 2346280 Zbl 1129.30031

[Series 2005] C. Series, "Limits of quasi-Fuchsian groups with small bending", Duke Math. J. 128:2 (2005), 285-329. MR 2006a:30043 Zbl 1081.30038 
[Wolpert 1979] S. Wolpert, "The length spectra as moduli for compact Riemann surfaces", Ann. of Math. (2) 109:2 (1979), 323-351. MR 80j:58067 Zbl 0441.30055

Received June 14, 2007. Revised February 25, 2008.

\section{YOUNG-EUN CHOI}

DePARTMENT OF MATHEMATICS AND STATistics

3000 IVYSIDE PARK

ALtOONA, PA 16601

UNITED STATES

choiye@psu.edu

http://math.aa.psu.edu/ choiye/

\section{KASRA RAFI}

DEPARTMENT OF MATHEMATICS

5734 S. UniVERsity AVENUE

ChICAgo, IL 60637

United STATES

rafi@math.uchicago.edu

http://www.math.uchicago.edu/ rafi/

\section{Caroline Series}

MATHEMATICS INSTITUTE

UNIVERSITY OF WARWICK

COVENTRY CV4 7AL

UNITED KINGDOM

c.m.series@warwick.ac.uk

http://www.warwick.ac.uk/ masbb/ 